Psychological Medicine, 1983, 13, 239-249

Printed in Great Britain

\title{
EDITORIAL
}

\section{Follow-up studies of anorexia nervosa: a review of research findings ${ }^{1}$}

There has been a recent spate of studies on the course of anorexia nervosa which call for collation, review and analysis. The practical value of such a synopsis is related to the fact that only by means of longitudinal investigations can the efficiency of treatment be judged. As long as the aetiology of the illness remains incompletely understood therapeutic measures must be symptomatic, and the outcome of the disease may reflect its natural history as well as therapeutic intervention. This survey represents the fruits of a systematic analysis of the available literature. It presents, first, the general aspects of follow-up studies, then their results, followed by factors influencing prognosis, and some implications for further research.

\section{GENERAL DESCRIPTION}

The follow-up reports analysed here are drawn from 45 studies in the English and German languages, published between 1953 and 1981. Their general characteristics are summarized in Table 1. In the main, the work has been concerned with two themes: the effectiveness of different methods of treatment, and the identification of prognostic factors. The number of patients examined varies between 6 and 140. Studies with the smallest number of patients (Niederhoff et al. 1975; Bhanji \& Thompson, 1974) deal with tests of the effectiveness of systematically-applied forms of treatment purely medical treatment and behaviour therapy. Theander (1970) and Dally (1969) studied 94 and 140 patients respectively; these larger numbers reflect the longer follow-up periods of the two investigations.

In order to gather evidence on the course of anorexia nervosa Morgan \& Russell (1975) make a plea for a follow-up of at least 4 years. They assume that anorexia nervosa has a rather long duration, so that improvement and recovery may take several years. An examination of the available studies revealed some findings which meet this contention and others which were obtained too near the termination of treatment to represent stable follow-up data (e.g. Tolstrup, 1965; Crisp, 1965, 1966; Bhanji \& Thompson, 1974; Brady \& Rieger, 1975). Longer follow-up periods were attained in the studies of Farquharson \& Hyland (1966), Theander (1970), Cremerius (1978) and Ziolko (1978).

Most of the data on the age of onset relate to the type of anorexia nervosa which begins in young adults, but a number of authors (Lesser et al. 1960; Blitzer et al. 1961; Tolstrup, 1965; Warren, 1968) have also examined patients whose illnesses began before puberty. Some workers have regarded the age of onset as a diagnostic criterion (e.g. Thomä, 1961; Dally \& Sargant, 1966; Ziegler \& Sours, 1968; Brady \& Rieger, 1975; Feighner et al. 1972), and it may be questioned whether patients whose symptoms appear first at the age of 40 (Morgan \& Russell, 1975) or even 59 (Seidensticker \& Tzagournis, 1968) qualify as genuine cases of anorexia nervosa.

As can be seen from Table 1, the diagnostic criteria which are employed in many studies are often incomplete or inadequately described. This hinders comparison between findings, since it is possible that different types of patient were included. Further, the variations in approach and diagnostic criteria of those studies with more comprehensive information render comparative assessment difficult. It would clearly be desirable to adopt diagnostic criteria which are relevant to anorexia

\footnotetext{
1 Address for correspondence: Professor H.-C. Steinhausen, Department of Child and Adolescent Psychiatry and Neurology, Free University of Berlin, Platanenallee 23, D-1000 Berlin 19, West Germany.
} 
Table 1. Sample characteristics of follow-up studies of anorexia nervosa

\begin{tabular}{|c|c|c|c|c|c|c|c|c|c|}
\hline \multirow[b]{3}{*}{ Study } & \multicolumn{2}{|c|}{ Sample size } & & & & & \multirow{3}{*}{$\begin{array}{l}\text { Age at } \\
\text { onset of } \\
\text { the } \\
\text { disease } \\
\text { (years) }\end{array}$} & \multirow{3}{*}{$\begin{array}{l}\text { Diagnostic } \\
\text { criteria }\end{array}$} & \multirow{3}{*}{$\begin{array}{c}\text { Therapeutic } \\
\text { measures }\end{array}$} \\
\hline & \multirow{2}{*}{$\begin{array}{l}\text { Patients } \\
\text { at } \\
\text { follow-up } \\
\text { (no.) }\end{array}$} & \multirow{2}{*}{$\begin{array}{l}\text { Drop- } \\
\text { out } \\
\text { rate } \\
(\%)\end{array}$} & \multicolumn{4}{|c|}{ Duration of follow-up (years) } & & & \\
\hline & & & I & II & III & IV & & & \\
\hline 1. Kay (1953) & 33 & $13 \cdot 1$ & $5->10$ & - & - & - & $16-20$ & $A N, A M, W L$ & PT, MT \\
\hline $\begin{array}{l}\text { 2. Beck \& Brachner- } \\
\text { Mortensen (1954) }\end{array}$ & 25 & $10 \cdot 7$ & - & - & $1-23$ & - & $11-31$ & $A N, A M, W L$ & MT \\
\hline 3. Williams (1958) & 42 & $14 \cdot 3$ & $3-21$ & - & - & - & $13-40^{*}$ & n.a. & MT \\
\hline 4. Lesser et al. (1960) & 15 & 0 & - & $1-17$ & - & - & $10-16$ & n.e.d. & $\mathrm{MT}$ \\
\hline 5. Thomä (1961) & 18 & $40 \cdot 0$ & - & - & $0 \cdot 6-7$ & - & $13-25$ & AN, AM, VO & PT \\
\hline 6. Blitzer et al. (1961) & 15 & n.a. & n.a. & - & - & - & $7-14$ & WL & PT, MT \\
\hline 7. Meyer (1961) & 20 & n.a. & - & - & $10-17$ & - & n.e.d. & n.a. & n.e.d. \\
\hline 8. Tolstrup (1965) & 28 & 0 & - & - & $0 \cdot 5-12$ & - & $7-24$ & n.a. & PT, MT \\
\hline 9. Frahm (1965) & 30 & 8.8 & - & - & 3 & - & $15-18$ & n.a. & MT \\
\hline 10. Frazier (1965) & 39 & n.a. & - & - & $5-20$ & - & 9-35 & $\begin{array}{l}\text { WL, HY, NMI } \\
\text { Distorted } \\
\text { body image }\end{array}$ & PT, MT \\
\hline 11. Kay \& Shapira (1965) & 60 & 7.7 & - & - & $3-10$ & - & $20-22$ & n.a. & MT \\
\hline 12. Crisp $(1965,1966)$ & 21 & n.a. & - & - & $0 \cdot 1-3 \cdot 6$ & - & n.a. & n.a. & PT, MT \\
\hline $\begin{array}{l}\text { 13. Farquharson \& } \\
\text { Hyland (1966) }\end{array}$ & 15 & $6 \cdot 2$ & - & - & $20-30$ & - & $13-23$ & n.a. & PT, MT \\
\hline 14. Dally \& Sargant (1966) & 57 & n.a. & - & - & - & $3-5$ & $17-19$ & $\begin{array}{l}\text { AN, AM, WL, } \\
\text { NMI, NPD }\end{array}$ & MT \\
\hline 15. Warren (1968) & 18 & 0 & $2 \cdot 6-11$ & - & - & - & $10-15$ & n.a. & PT, MT \\
\hline $\begin{array}{l}\text { 16. Seidensticker \& } \\
\text { Tzagournis (1968) }\end{array}$ & 53 & $11 \cdot 7$ & - & - & - & $1->10$ & $10-59$ & $\begin{array}{l}\text { AN, WL, } \\
\text { NMI, NPD }\end{array}$ & PT, MT \\
\hline $\begin{array}{l}\text { 17. Browning \& Miller } \\
\text { (1968) }\end{array}$ & 36 & n.a. & $2-32$ & - & - & - & n.a. & $\begin{array}{l}\text { AN, WL, } \\
\text { NMI, NPD }\end{array}$ & PT, MT \\
\hline 18. Ziegler \& Sours (1968) & 26 & $77 \cdot 4$ & - & - & $1-34$ & - & $10-15$ & AN, WL, NMI & n.a. \\
\hline 19. Datly (1969) & 140 & 0 & - & - & - & $<17$ & $11-33$ & AN, AM, WL & MT \\
\hline 20. Theander (1970) & 94 & $2 \cdot 1$ & - & - & - & 6 & $11-34$ & $\begin{array}{l}\text { Disturbed } \\
\text { eating attitude } \\
\text { and body image }\end{array}$ & PT, MT \\
\hline 21. Valanne el al. (1972) & 30 & $\mathbf{0}$ & - & - & $1-15$ & - & $11-16$ & $\begin{array}{l}\text { AN, AM, NMI, } \\
\text { NPD }\end{array}$ & PT, MT \\
\hline 22. Halmi et al. (1973) & 36 & $14 \cdot 3$ & $1-30$ & - & - & $-<$ & $\begin{array}{l}15(N=13) \\
15(N=23)\end{array}$ & $\begin{array}{l}\text { Feighner el al. } \\
\text { criteria }\end{array}$ & n.a. \\
\hline 23. Bruch (1973) & 38 & $13 \cdot 3$ & $1-19$ & - & - & - & $20-26$ & $\begin{array}{l}\text { Distorted } \\
\text { body image }\end{array}$ & PT, MT \\
\hline $\begin{array}{l}\text { 24. Bhanji \& Thompson } \\
\text { (1974) }\end{array}$ & 7 & $63 \cdot 6$ & - & - & - & $0 \cdot 2-6$ & $14-34^{*}$ & n.a. & BT, MT \\
\hline 25. Niskanen et al. (1974) & 48 & n.a. & - & - & $1-18$ & - & $13-32$ & $\begin{array}{l}\text { AN, WL, NMI, } \\
\text { NPD }\end{array}$ & PT, MT \\
\hline 26. Silverman (1974) & 27 & 6.9 & - & - & - & 6 & $9-15$ & n.a. & PT, MT \\
\hline $\begin{array}{l}\text { 27. Morgan \& Russell } \\
\text { (1975) }\end{array}$ & 41 & 0 & - & - & $>4$ & - & $11-40$ & $\begin{array}{l}\text { AN, AM, WL, } \\
\text { HY, LA }\end{array}$ & PT \\
\hline 28. Brady \& Rieger (1975) & 15 & $6 \cdot 2$ & - & - & $0.4-4$ & - & $15-34^{*}$ & $\mathrm{AM}, \mathrm{WL}$ & BT \\
\hline 29. Pierloot et al. (1975) & 32 & 0 & - & - & - & $1-6$ & $12-25+$ & $\begin{array}{l}\text { AN, AM, WL, } \\
\text { NMI, NPD }\end{array}$ & PT, BT, MT \\
\hline 30. Niederhoff et al. (1975) & 6 & 0 & - & - & - & $1-4$ & $11-15$ & n.a. & MT \\
\hline 31. Beumont et al. (1976) & 31 & n.a. & - & - & - & $0 \cdot 3-2$ & Mean $=17$ & $\begin{array}{l}\text { AN, WL, NMI, } \\
\text { NPD }\end{array}$ & n.a. \\
\hline 32. Halmi et al. (1976) & 79 & $16 \cdot 8$ & $1-50$ & - & - & - & $\begin{array}{c}<25 \\
(N=69)\end{array}$ & $\begin{array}{l}\text { Feighner et al. } \\
\text { criteria }\end{array}$ & n.a. \\
\hline 33. Willi \& Hagemann (1976) & 20 & 0 & - & $\underset{11}{\text { Mean }}=$ & - & - & $\begin{array}{c}\text { Mean }= \\
19.5\end{array}$ & $\begin{array}{l}\text { AN, AM, WL } \\
\text { (VO, HY, LA, } \\
\text { BU) }\end{array}$ & PT, MT \\
\hline 34. Rosman et al. (1976) & 53 & 0 & - & - & - & $0 \cdot 3-4$ & $9-21$ & $\begin{array}{l}\text { AN, distorted } \\
\text { body image }\end{array}$ & FT \\
\hline 35. Goetz et al. (1977) & 30 & 0 & - & $5-20$ & - & - & $9-16^{*}$ & $\begin{array}{l}\text { WL, distorted } \\
\text { body image, NMI }\end{array}$ & PT \\
\hline $\begin{array}{l}\text { 36. Sturzenberger et al. } \\
\text { (1977), Cantwell et al. } \\
\text { (1977) }\end{array}$ & 26 & $21 \cdot 2$ & - & - & - & $\underset{4 \cdot 9}{\text { Mean }}=$ & $11-16$ & $\begin{array}{l}\text { Feighner et al. } \\
\text { criteria }\end{array}$ & $\begin{array}{l}\text { In-patient } \\
\text { treatment }\end{array}$ \\
\hline 37. Stonehill \& Crisp (1977) & 38 & $13 \cdot 3$ & - & - & - & $4-7$ & $\underset{20^{*}}{\operatorname{Mean}}=$ & n.a. & PT, MT \\
\hline
\end{tabular}


Table 1. (cont.)

\begin{tabular}{|c|c|c|c|c|c|c|c|c|c|}
\hline \multirow[b]{3}{*}{ Study } & \multicolumn{2}{|c|}{ Sample size } & & & & & \multirow{3}{*}{$\begin{array}{c}\text { Age at } \\
\text { onset of } \\
\text { the } \\
\text { disease } \\
\text { (years) }\end{array}$} & \multirow{3}{*}{$\begin{array}{l}\text { Diagnostic } \\
\text { criteria }\end{array}$} & \multirow{3}{*}{$\begin{array}{l}\text { Therapeutic } \\
\text { measures }\end{array}$} \\
\hline & \multirow{2}{*}{$\begin{array}{l}\text { Patients } \\
\text { at } \\
\text { follow-up } \\
\text { (no.) }\end{array}$} & \multirow{2}{*}{$\begin{array}{c}\text { Drop- } \\
\text { out } \\
\text { rate } \\
(\%)\end{array}$} & \multicolumn{4}{|c|}{ Duration of follow-up (years) } & & & \\
\hline & & & I & II & III & IV & & & \\
\hline 38. Garfinkel et al. (1977) & 42 & n.a. & Mean $=5$ & - & - & - & $11-20$ & $\begin{array}{l}\text { Feighner et al. } \\
\text { criteria }\end{array}$ & $\begin{array}{l}\mathrm{PT},(\mathrm{FT}, \mathrm{BT}) \\
\mathrm{MT}\end{array}$ \\
\hline 39. Pertschuk (1977) & 27 & 6.9 & - & - & - & $0 \cdot 3-3 \cdot 9$ & $14-34^{*}$ & $\begin{array}{l}\text { Feighner et al. } \\
\text { criteria }\end{array}$ & BT, MT \\
\hline 40. Cremerius (1978) & 11 & $15 \cdot 4$ & - & - & - & $26-29$ & Puberty & n.a. & PT, MT \\
\hline 41. Ziolko (1978) & 28 & 0 & - & - & - & $5-20$ & $14-18$ & n.a. & PT, MT \\
\hline 42. Petzold (1979) & 44 & $4 \cdot 5$ & - & - & - & $\underset{2.7}{\text { Mean }}=$ & $13-29$ & n.e.d. & PT (FT), MT \\
\hline 43. Hsu et al. (1979) & 100 & $2 \cdot 8$ & - & - & $1-18$ & - & $13-32$ & n.e.d. & PT, MT \\
\hline 44. Schütze (1980) & 49 & $22 \cdot 3$ & - & - & - & $0 \cdot 3-8 \cdot 6$ & Puberty & n.a. & PT, MT \\
\hline 45. Rollins \& Piazza (1981) & 35 & $50 \cdot 0$ & - & - & $>2$ & - & $\begin{array}{c}\text { Mean }= \\
13 \cdot 7\end{array}$ & $\begin{array}{l}\text { WL, weight } \\
\text { phobia, distorted } \\
\text { body image }\end{array}$ & n.a. \\
\hline
\end{tabular}

I, related to onset of the disease; II, related to onset of therapy; III, related to termination of therapy; IV, starting point not described; - at hospital admission: $\nmid$ at termination.

$A N=$ anorexia; $A M=$ amenorrhoea; $W L=$ weight loss; $V O=$ vomiting; $H Y=$ hyperactivity; $L A=$ laxative abuse; $B U=$ bulimia; $\mathrm{NMI}=$ no known medical illness; NPD = no other known psychiatric disorder.

BT = behaviour therapy; FT = family therapy; $M T=$ medical treatment; $P T=$ psychotherapy; .a. $=$ not assessed; n.e.d. $=$ not exactly described.

as a syndrome and also find general acceptance in clinical practice. Despite much criticism (Andersen, 1977; Fries, 1977; Rollins \& Piazza, 1978), these desiderata appear to be best fulfilled by the criteria of Feighner et al. (1972).

Treatment regimes of internal medicine and of psychotherapy are included in the follow-up studies under survey. The two types of procedure are mostly used in combination, seldom individually. With only a few exceptions (Dally \& Sargant, 1966; Rosman et al. 1976; Garfinkel et al. 1977; Petzold, 1979), the studies on the effectiveness of certain forms of treatment employed smaller patient-samples than those which concentrated on prognostic factors. This difference may have something to do with a lack of sufficient subjects on whom a particular therapeutic procedure could be systematically carried out. The psychotherapeutic procedures of behaviour therapy and family therapy have been applied with increasing frequency in recent years.

The extent to which treatment programmes exert a long-term positive influence on the course of anorexia nervosa is assessed differently by various workers. On the basis of their follow-up results, several authors support the view that there is neither a specifically effective form of treatment for anorexia nervosa, nor any qualitative differences between therapeutic regimes (Kay, 1953; Frazier, 1965; Browning \& Miller, 1968; Theander, 1970; Morgan \& Russell, 1975; Bhanji \& Thompson, 1974; Garfinkel et al. 1977; Cremerius, 1978). Others, however, have attempted to prove the effectiveness of their methods of treatment by reference to their follow-up results (e.g. Frahm, 1965; Dally \& Sargant, 1966; Rosman et al. 1976; Niederhoff et al. 1975; Petzold, 1979). Most.of the findings presented in these studies should be viewed critically. Either they involved too few subjects; or the follow-up periods were too short; or the evaluation of response was based on too small a number of criteria.

\section{FOLLOW-UP RESULTS}

Because of the difficulty of collecting objective information from samples of anorexic subjects, most workers appear to have limited themselves to the collection of more subjective data, basing their investigations on material obtained from former patients, supplemented in part by relatives and doctors, without employing standardized questionnaires or interviews by an independent observer. Such a method of investigation must considerably distort follow-up results and lessen their value 
Table 2. Results of follow-up-studies of anorexia nervosa

\begin{tabular}{|c|c|c|c|c|c|c|c|c|}
\hline \multirow[b]{2}{*}{ Study } & \multicolumn{3}{|c|}{ Normalization of } & \multirow[b]{2}{*}{$\begin{array}{l}\text { Psychiatric } \\
\text { status } \\
(\%)\end{array}$} & \multirow[b]{2}{*}{$\begin{array}{c}\text { Psychosocial } \\
\text { adaptation } \\
(\%)\end{array}$} & \multirow[b]{2}{*}{$\begin{array}{c}\text { Chron- } \\
\text { icity } \\
(\%)\end{array}$} & \multirow[b]{2}{*}{$\begin{array}{l}\text { Mortality } \\
\text { rate } \\
(\%)\end{array}$} & \multirow[b]{2}{*}{$\begin{array}{l}\text { Improvement } \\
\text { rate } \\
(\%)\end{array}$} \\
\hline & $\begin{array}{l}\text { Weight } \\
(\%)\end{array}$ & $\begin{array}{l}\text { Men- } \\
\text { struation } \\
(\%)\end{array}$ & $\begin{array}{c}\text { Eating } \\
\text { behav- } \\
\text { iour } \\
(\%)\end{array}$ & & & & & \\
\hline 1. Kay (1953) & $\sim 50$ & 55 & 35 & $\begin{array}{l}\text { DS }=6 \\
\text { NS }=40 \\
\text { OS }=6\end{array}$ & $\begin{array}{l}\text { Family }=9 \\
\text { Occupation }=3\end{array}$ & 21 & 18 & $\begin{array}{l}r=12 \\
i=48\end{array}$ \\
\hline $\begin{array}{l}\text { 2. Beck \& Brachner- } \\
\text { Mortensen (1954) }\end{array}$ & 64 & 68 & 68 & n.a. & $\begin{array}{l}\text { Marriage }=60 \\
\text { Children }=52\end{array}$ & 16 & 4 & $\begin{array}{l}\text { 'Excellent } \\
\text { health' }=80\end{array}$ \\
\hline 3. Williams (1958) & 55 & n.a. & n.a. & n.a. & $\begin{array}{l}\text { Marriage }=28 \\
\text { Children }=14 \\
\text { Occupation }=55\end{array}$ & 7 & 19 & $\begin{array}{l}r=55 \\
i=14\end{array}$ \\
\hline 4. Lesser et al. (1960) & 60 & n.a. & n.a. & $\begin{array}{l}H P=53 \\
O P=27 \\
S P=20\end{array}$ & Good $/$ fair $=87$ & 13 & 0 & Good $/$ fair $=87$ \\
\hline 5. Thomä (1961) & 36 & 73 & 41 & n.a. & $\begin{array}{l}\text { Marriage }=5 \\
\text { Children }=9 \\
\text { Occupation }=45\end{array}$ & 13 & 3 & $r / i=33$ \\
\hline 6. Blitzer et al. (1961) & n.a. & n.a. & 60 & $D P=87$ & $\begin{array}{l}\text { Improvement } \\
\text { in general per- } \\
\text { sonality }=60\end{array}$ & 0 & 7 & $\begin{array}{l}r=60 \\
i=33\end{array}$ \\
\hline 7. Meyer (1961) & n.e.d. & 90 & 35 & $\begin{array}{l}H P=15 \\
O P=75 \\
S C=15\end{array}$ & n.a. & 35 & 15 & $\mathrm{i}=35$ \\
\hline 8. Tolstrup (1965) & n.a. & n.a. & n.a. & n.a. & Marriage $=18$ & 14 & 0 & $\begin{array}{l}\text { Good }=22 \\
\text { Fair }=63\end{array}$ \\
\hline 9. Frahm (1965) & n.e.d. & 67 & 97 & n.a. & 100 & 0 & 0 & $\sim 100$ \\
\hline 10. Frazier (1965) & n.a. & n.a. & 28 & $\mathrm{SC}=28$ & $\begin{array}{l}\text { Occupational/ } \\
\text { social relation- } \\
\text { ships }=28\end{array}$ & 31 & 8 & $\begin{array}{l}\text { Markedly } i=28 \\
\text { Slightly } i=33\end{array}$ \\
\hline 11. Kay \& Shapira (1965) & $\sim 50$ & $\sim 40$ & $\sim 40$ & $\begin{array}{l}\text { DS/OS }=27 \\
N S=34 \\
S C=3\end{array}$ & Marriage $=34$ & 27 & 17 & $\begin{array}{l}\text { Markedly } i=34 \\
\text { Partly } i=34\end{array}$ \\
\hline 12. Crisp $(1965,1966)$ & 71 & 44 & 52 & DS $=4$ & $\begin{array}{l}\text { Marriage }=28 \\
\text { Sexuality }=9\end{array}$ & n.e.d. & 9 & 43 \\
\hline $\begin{array}{l}\text { 13. Farquharson \& } \\
\text { Hyland (1966) }\end{array}$ & n.e.d. & 83 & 87 & $\begin{array}{l}\text { NS }=20 \\
S C=7\end{array}$ & 67 & 7 & 0 & $r=67$ \\
\hline 14. Dally \& Sargant (1966) & $60-72$ & $69-72$ & $55-67$ & $\begin{array}{l}\mathrm{DS}=3 \\
\mathrm{OS}=3 \\
\mathrm{SC}=3 \\
\text { Psychopathy }=6 \\
\text { Phobias }=3-7\end{array}$ & $\begin{array}{l}\text { Marriage }=33-41 \\
\text { Occupation }=6-15 \\
\text { Good }=60-72\end{array}$ & $\sim 3-6$ & n.a. 6 & $60-72$ \\
\hline 15. Warren (1968) & 55 & 55 & 61 & $\begin{array}{l}\mathrm{DS}=5 \\
\mathrm{NS}=39 \\
\mathrm{SC}=5\end{array}$ & $\begin{array}{l}\text { Marriage }=5 \\
\text { Sexuality }=28\end{array}$ & 28 & 11 & 61 \\
\hline $\begin{array}{l}\text { 16. Seidensticker \& } \\
\text { Tzagournis (1968) }\end{array}$ & 63 & 69 & n.e.d. & n.a. & $\begin{array}{l}\text { 'Active and } \\
\text { productive } \\
\text { life' }=38\end{array}$ & 31 & 13 & $\begin{array}{l}\text { Fair }=38 \\
\text { Partly fair }=30\end{array}$ \\
\hline 17. Browning \& Miller (1968) & 75 & $\sim 50$ & 50 & n.a. & n.a. & 16 & 8 & $\begin{array}{l}i=50 \\
\text { Moderately } i=25\end{array}$ \\
\hline $\begin{array}{l}\text { 18. Ziegler \& Sours (1968) } \\
\text { 19. Dally (1969) }\end{array}$ & $\begin{array}{c}\text { n.e.d. } \\
69\end{array}$ & $\begin{array}{c}\text { n.e.d. } \\
59\end{array}$ & $\begin{array}{l}\text { n.e.d. } \\
\text { n.e.d. }\end{array}$ & $\begin{array}{l}\text { n.a. } \\
\text { DS }=24 \\
\text { OS }=11 \\
\text { SC }=1 \\
\text { Phobias = 10 } \\
\text { Hypochon- } \\
\text { driasis }=23\end{array}$ & $\begin{array}{l}\text { Marriage }=46 \\
\text { Marriage }=31 \\
\text { Occupation }=25\end{array}$ & $\begin{array}{l}11 \\
29\end{array}$ & $\begin{array}{l}5 \\
3\end{array}$ & $\begin{array}{l}\text { n.e.d. } \\
69\end{array}$ \\
\hline 20. Theander (1970) & 63 & 76 & 63 & $\begin{array}{l}\mathrm{DS}=29 \\
O S=13 \\
\mathrm{SC}=1 \\
\text { Anxiety }=13\end{array}$ & Marriage $=50$ & 7 & 10 & $\mathrm{i}=63$ \\
\hline 21. Valanne et al. (1972) & n.e.d. & n.e.d. & 47 & $\begin{array}{l}\mathrm{NS}=27 \\
\mathrm{PS}=13\end{array}$ & $\begin{array}{l}\text { Marriage }=13 \\
\text { Children }=7 \\
\text { Occupation }=90 \\
\text { Independence } \\
\text { from the } \\
\text { family = } 23\end{array}$ & 17 & 0 & $\begin{array}{l}\text { Free of } \\
\text { symptoms }=10\end{array}$ \\
\hline 22. Halmi et al. (1973) & 41 & 55 & n.e.d. & $\begin{array}{l}\mathrm{DS}=27 \\
\mathrm{NS}=61\end{array}$ & $\begin{array}{l}\text { Marriage }=34 \\
\text { Occupation }=36 \\
\text { Sexuality }=32\end{array}$ & 14 & 16 & $\begin{array}{l}r=25 \\
i=23 \\
\text { Moderately } i=23\end{array}$ \\
\hline
\end{tabular}


Table 2. (cont.)

\begin{tabular}{|c|c|c|c|c|c|c|c|c|}
\hline \multirow[b]{2}{*}{ Study } & \multicolumn{3}{|c|}{ Normalization of } & \multirow[b]{2}{*}{$\begin{array}{l}\text { Psychiatric } \\
\text { status } \\
(\%)\end{array}$} & \multirow[b]{2}{*}{$\begin{array}{l}\text { Psychosocial } \\
\text { adaptation } \\
\text { (\%) }\end{array}$} & \multirow[b]{2}{*}{$\begin{array}{l}\text { Chron- } \\
\text { icity } \\
(\%)\end{array}$} & \multirow[b]{2}{*}{$\begin{array}{c}\text { Mortality } \\
\text { rate } \\
(\%)\end{array}$} & \multirow[b]{2}{*}{$\begin{array}{l}\text { Improvement } \\
\text { rate } \\
(\%)\end{array}$} \\
\hline & $\begin{array}{l}\text { Weight } \\
(\%)\end{array}$ & $\begin{array}{l}\text { Men- } \\
\text { struation } \\
(\%)\end{array}$ & $\begin{array}{c}\text { Eating } \\
\text { behav- } \\
\text { iour } \\
(\%)\end{array}$ & & & & & \\
\hline 23. Bruch (1973) & 61 & n.a. & $\sim 33$ & $S C=10$ & 33 & 13 & 8 & $r=33$ \\
\hline 24. Bhanji \& Thompson (1974) & 43 & 28 & 57 & n.a. & n.a. & 43 & 0 & $\begin{array}{l}\text { Good }=14 \\
\text { Fair }=43\end{array}$ \\
\hline 25. Niskanen et al. (1974) & n.e.d. & n.a. & n.e.d. & $\begin{array}{l}\mathrm{BS}=2 \\
\mathrm{NS}=57\end{array}$ & $\begin{array}{l}\text { Marriage }=12 \\
\text { Children }=10 \\
\text { Occupation }=48\end{array}$ & n.a. & 8 & 42 \\
\hline 26. Silverman (1974) & n.e.d. & n.e.d. & n.e.d. & $S C=15$ & n.a. & 18 & 0 & $\begin{array}{l}\text { 'Well } \\
\text { functioning' }=33\end{array}$ \\
\hline $\begin{array}{l}\text { 27. Morgan \& Russell } \\
\text { (1975) }\end{array}$ & 68 & 50 & 33 & $\begin{array}{l}\text { DS }=45 \\
\text { OS }=23\end{array}$ & $\begin{array}{l}\text { Occupation }=73 \\
\text { Sexuality }=60\end{array}$ & 29 & $5^{a}$ & $\begin{array}{l}\text { Good }=39 \\
\text { Fair }=27\end{array}$ \\
\hline 28. Brady \& Rieger (1975) & 77 & 36 & n.a. & n.a. & $\begin{array}{l}\text { Family/ } \\
\text { occupation/ } \\
\text { social relation- } \\
\text { ships: } \operatorname{good}=38\end{array}$ & 23 & 7 & $\begin{array}{l}\text { Good }=38 \\
i=38\end{array}$ \\
\hline 29. Pierloot et al. (1975) & 50 & 50 & 50 & n.e.d. & $\begin{array}{l}\text { Good social } \\
\text { functioning }=50\end{array}$ & 34 & 0 & $\begin{array}{l}r=50 \\
i=16\end{array}$ \\
\hline 30. Niederhoff et al. (1975) & 66 & 66 & 66 & $\mathrm{SP}$ and $\mathrm{OS}=50$ & $\begin{array}{l}\text { Normalization } \\
\text { of life }=83\end{array}$ & 16 & 0 & $\begin{array}{l}\text { Good }=66 \\
\text { Fair }=16\end{array}$ \\
\hline 31. Beumont et al. (1976) & $41^{\circ}$ & n.a. & $41^{\circ}$ & $\mathrm{HP}^{b}=6, \mathrm{OP}^{b}=76$ & n.a. & $29^{b}$ & 0 & $41^{\circ}$ \\
\hline & $21^{c}$ & n.a. & $21^{c}$ & $H P^{c}=50, O P^{c}=57$ & n.a. & $79^{c}$ & 0 & $21^{c}$ \\
\hline 32. Halmi et al. (1976) & n.a. & n.a. & n.a. & $\begin{array}{l}E D=2 \\
S C=1 \\
H P=4\end{array}$ & n.a. & 11 & 21 & $r=51$ \\
\hline 33. Willi \& Hagemann (1976) & 15 & 63 & 31 & $\begin{array}{l}\mathrm{ED}=5 \\
\mathrm{DS}=50 \\
\mathrm{SC}=5\end{array}$ & Marriage $=60$ & 25 & 5 & $\begin{array}{l}r=35 \\
i=35\end{array}$ \\
\hline 34. Rosman et al. (1976) & n.a. & n.a. & n.a. & n.a. & $\begin{array}{l}\text { Family/school/ } \\
\text { occupation: } \\
\text { Good }=88\end{array}$ & 6 & 0 & $\begin{array}{l}r=86 \\
i=8\end{array}$ \\
\hline 35. Goetz et al. (1977) & 87 & 92 & n.e.d. & $\begin{array}{l}\mathrm{HP}=47 \\
\mathrm{OP}=40 \\
\mathrm{SC}=13\end{array}$ & Good $=60$ & 17 & 0 & $\begin{array}{l}\text { Good }=60 \\
\text { Fair }=23\end{array}$ \\
\hline $\begin{array}{l}\text { 36. Sturzenberger et al. } \\
\text { (1977), Cantwell et al. } \\
\text { (1977) }\end{array}$ & $\begin{array}{l}77^{d} \\
83^{\circ}\end{array}$ & $\begin{array}{l}70^{d} \\
83^{e}\end{array}$ & $\begin{array}{l}81^{d} \\
83^{e}\end{array}$ & $\begin{array}{l}\text { DS }=67^{d}, 56^{e} \\
\text { OS }=19^{d}, 44^{e} \\
\text { Phobias }=7^{d}, 6^{e}\end{array}$ & $\begin{array}{l}\text { Occupation/ } \\
\text { social relation- } \\
\text { ships/sexuality: } \\
\text { good }=46^{d}, 67^{\circ}\end{array}$ & n.e.d. & 0 & Good $=67$ \\
\hline 37. Stonehill \& Crisp (1977) & 68 & 68 & n.a. & n.e.d. & Good/fair $=71$ & 7 & n.e.d. & 68 \\
\hline 38. Garfinkel et al. (1977) & 58 & 49 & 29 & n.a. & $\begin{array}{l}\text { Good }=7 \\
\text { Fair }=41 \\
\text { Occupation }=49\end{array}$ & n.a. & $2 i$ & $\mathrm{i}=50$ \\
\hline 39. Pertschuk (1977) & 67 & 37 & n.e.d. & $\mathrm{DS}=7$ & $\begin{array}{l}\text { Family/ } \\
\text { occupation/ } \\
\text { social relation- } \\
\text { ships: } \\
\text { Good }=44\end{array}$ & 22 & 0 & $r=7$ \\
\hline 40. Cremerius (1978) & n.e.d. & n.e.d. & n.e.d. & $\begin{array}{l}N S=9 \\
P S=36\end{array}$ & $\begin{array}{l}\text { Occupation }=45 \\
\text { Sexuality }=18\end{array}$ & 36 & 18 & 45 \\
\hline 41. Ziolko (1978) & n.e.d. & 96 & $\sim 50$ & $\begin{array}{l}\mathrm{DS}=25 \\
\mathrm{PS}=3\end{array}$ & $\begin{array}{l}\text { Marriage }=35 \\
\text { Occupation }=89\end{array}$ & 25 & 3 & $\begin{array}{l}r=60 \\
i=28\end{array}$ \\
\hline 42. Petzold (1979) & n.e.d. & 25 & 54 & n.a. & $\begin{array}{l}\text { School/ } \\
\text { occupation/ } \\
\text { social relation- } \\
\text { ships }=61\end{array}$ & 16 & 11 & $\begin{array}{l}r=27 \\
i=45\end{array}$ \\
\hline 43. Hsu et al. (1979) & 64 & 54 & 37 & $O S=22$ & $\begin{array}{l}\text { Marriage }=29 \\
\text { Children }=14 \\
\text { Sexuality }=83 \\
\text { Occupation }=82\end{array}$ & 20 & 2 & Good $=48$ \\
\hline 44. Schütze (1980) & 57 & 57 & 57 & n.a. & 57 & $21 \cdot 5$ & 0 & $\mathrm{i}=57$ \\
\hline 45. Rollins \& Piazza (1981) & 65 & 64 & n.e.d. & n.e.d. & 79 & 14 & 0 & $r / i=69$ \\
\hline
\end{tabular}

BS = borderline symptoms; $D S=$ depressive symptoms; NS = neurotic symptoms; OS = obsessive-compulsive symptoms; ED $=$ endogenous depression; $\mathrm{PS}=$ psychosis; $\mathrm{SC}=$ schizophrenia; $\mathrm{DP}=$ depressive personality; $H \mathrm{P}=$ hysterical personality; $\mathrm{OP}=$ obsessional personality; $\mathbf{S P}=$ schizoid personality.

a Including $N=2$ patients with death due to other causes.

- Patients with weight loss due to diet, food refusal, and exercising.

c Patients with weight loss due to habitual vomiting and abuse of purgatives.

d Information obtained by the parents. 
unless the patients' veracity can be established. Very few authors used standardized tests in their follow-up investigations (e.g. Seidensticker \& Tzagournis, 1968; Browning \& Miller, 1968; Theander, 1970; Stonehill \& Crisp, 1977; Sturzenberger et al. 1977). The principal results are summarized in Table 2.

With regard to weight restoral, there was insufficient information in many of the papers, partly because relatively few of the authors appear to have included weight as a factor to be studied (e.g. Blitzer et al. 1961; Halmi et al. 1976). In some investigations the weight of former patients is recorded, but conclusions concerning subsequent weight-levels are limited by ambiguity (Tolstrup, 1965; Farquharson \& Hyland, 1966), an absence of exact data (Rosman et al. 1976) or a restriction of measurement to the average weight gain (Ziolko, 1978; Petzold, 1979). A large majority of the studies establish a weight restoral of between 50 and $70 \%$, and some present increases of between 70 and $80 \%$. Such good results are more often observed in patients whose illnesses began at an early age (Sturzenberger et al. 1977; Rosman et al. 1976; Warren, 1968). The lowest figure of 15\% is reported in the study by Willi \& Hagemann (1976), who recorded weight restoral in only 3 of their 20 subjects, while 10 of them were described as 'stably but slightly underweight'. The mean weight of patients examined after treatment was $21 \%$ below average.

Goetz et al. (1977) reported the highest figure: at the time of their follow-up 26 patients of their total of $30(87 \%)$ had a normal body weight, though this was not precisely defined. In relation to other assessments this statistic takes on a different significance. In terms of psychosocial adjustment, for example, $60 \%$ of the patients did well. Weight restoral alone cannot therefore be taken as sufficient evidence of recovery from anorexia nervosa. Only after a number of factors have been assessed can an assessment of the course of anorexia nervosa be properly formulated.

Amenorrhoea is widely held to be an essential diagnostic criterion in female patients from puberty onwards. A normalization of menstrual function is therefore seen as an index of recovery or improvement. As with weight restoral, most studies report rates of normalization between 50 and $70 \%$; an early onset of the disease was associated with better rates of improvement. This suggests that the criteria of change are inter-related, since a return of menstruation is usually dependent on a satisfactory weight-level (Dally, 1969). Higher rates of normalization are reported by Meyer (1961), Farquharson \& Hyland (1966), Goetz et al. (1977) and Ziolko (1978). Bhanji \& Thompson (1974), Beumont et al. (1976) and Petzold (1979) all present lower figures. Inspection of the studies reveals further that the higher rates are associated with longer follow-up periods, and the lower percentages with shorter periods. This correlation can be interpreted as suggesting that longer periods of observation increase the probability of a normalization of menstrual functioning.

The assessment of the normalization of eating disorders is particularly difficult when based on information derived from former patients, to whom food is a topic charged with emotion. This may partly explain the wide fluctuation of between 30 and $70 \%$ in published studies.

The best results (97\% of normalization) were achieved by Frahm (1965), who employed purely medical treatment. It is not, however, possible to judge whether this figure would have remained at this level after a follow-up period longer than 3 years. The poorest result $(21 \%)$ is reported by Beumont et al. (1976), who studied a group of patients among whom purgative abuse and vomiting were especially prominent symptoms. Long-term improvements of 20-50\% emerge from most follow-up reports, but it was not possible to deduce the extent to which a return to normal eating patterns exerted a positive effect on weight and menstruation. The findings suggest a tendency towards no more than a limited interaction. Willi \& Hagemann (1976), for example, report that while $31 \%$ of their patients showed normal eating patterns and $63 \%$ achieved regular menstruation, only $15 \%$ demonstrated weight restoral.

The varying forms of psychiatric diagnosis associated with anorexia nervosa make it difficult to draw general conclusions. Depression and obsessive-compulsive states or personality structure assume a prominent place in many studies. The frequency of depressive symptoms or personality traits fluctuates between 3 and $87 \%$, with an average of $31 \%$. The highest estimate of $87 \%$ comes from Blitzer et al. (1961), though the patients in this study were very young. In most studies depressive symptoms were recorded with moderate frequency (e.g. Halmi et al. 1973; Morgan \& 
Russell, 1975; Willi \& Hagemann, 1976; Sturzenberger et al. 1977) or rarely (Kay, 1953; Crisp, 1965; 1966; Dally \& Sargant, 1966; Warren, 1968; and Pertschuk, 1977). Similar differences, ranging from $3 \%$ to $83 \%$, are reported for the frequency of obsessive-compulsive symptoms and personality traits associated with anorexia nervosa. Sturzenberger et al. (1977), Cantwell et al. (1977), Beumont et al. (1976) and Meyer (1961) all furnish high estimates, while the lowest come again from Dally \& Sargant (1966) and Kay (1953).

The development of a psychotic disorder in cases of anorexia nervosa is recorded by most authors as occurring in fewer than $10 \%$ of cases, though some studies provide a higher estimate (Meyer, 1961 ; Frazier, 1965; Silverman, 1974; Goetz et al. 1977; Cremerius, 1978). The older notion that anorexia nervosa was in some instances a form of schizophrenic disorder has been largely abandoned. On the basis of present knowledge, the emergence of a psychotic state appears to be a separate condition which develops independently, and the possibility of mis-diagnosis cannot be excluded in some cases.

The effects of anorexia nervosa extend to the patient's social environment. The relationship to the social environment therefore becomes another factor in the assessment of recovery, though again it is difficult to obtain relevant data on the subject's behaviour in school, at work, in the family, in marriage, on their attitudes to sexuality and on their social contacts. Although it is relatively easy to assess scholastic, occupational and mental performance it is more difficult to evaluate the quality of social contacts, especially when the criteria are ill defined. Some of the information must therefore be treated with reservation. Furthermore, the varied forms in which the information is presented compounds the difficulty of integrating the studies with the aim of reaching general conclusions. In studies which grade their findings 'good-fair-poor', the most common estimate of recovery is $50-80 \%$, with younger patients exhibiting the better rates of improvement (Sturzenberger et al. 1977; Rosman et al. 1976). If other studies are included, however, then the figure of 50-80\% appears to be too high.

According to Dally \& Sargant (1966), patients can be said to have reached a condition of chronicity if they continue to display symptoms after 5 years despite suitable treatment. The rates of cases becoming chronic were less than $20 \%$ in the studies reviewed, and some workers report no chronicity (Blitzer et al. 1961; Frahm, 1965). These latter findings, however, are probably atypical: Blitzer et al., for example, included samples of very young patients; and Frahm's follow-up period of 3 years was too short. The highest figure for chronicity $(79 \%)$ is provided by Beumont $e t$ al. whose patients achieved weight reduction by means of vomiting and purgative abuse. The rate of less than $20 \%$ chronicity cited by the majority of studies differs from the much-quoted observation that anorexia nervosa is a chronic disease in one third of cases (Meyer, 1961; Willi \& Hagemann, 1976; Cremerius, 1978).

The mortality rate for anorexia nervosa has been calculated as 7-15\% (Sours, 1969). Among the studies under review there was a range of $0-21 \%$, the highest figure coming from Halmi et al. (1976). The two largest sub-groups were those reporting no mortality, followed closely by those with a mortality rate of less than $10 \%$.

Estimates of the rate of improvement are dependent on the criteria adopted. Some workers emphasize psychological adjustment, without reference to specifically anorexic symptoms (e.g. Lesser et al. 1960); others prefer the presence or absence of clinical symptoms (e.g. Thomä, 1961); all too often, no information is provided on the criteria employed (Blitzer et al. 1961). Some workers also demand undisturbed social behaviour or the full restitution of mental health (e.g. Tolstrup, 1965).

In view of such diverse criteria, it is not surprising that the published rates of improvement vary between 10 and $86 \%$, the majority falling between 30 and $50 \%$. The assumption of a one third improvement, however, appears to represent an underestimate, just as the assumption of a one third chronicity rate is excessive. High rates of improvement are reported by Frahm (1965), Lesser $e t$ al. (1960), who relied largely on medical treatment, and Rosman et al. (1976) for whom family therapy was the mainstay. 


\section{PROGNOSTIC FACTORS}

One of the chief aims of carrying out follow-up studies in the study of anorexia nervosa lies in the identification of possible prognostic factors, favourable or unfavourable. The more important are listed below.

\section{(a) Age of onset}

Most workers associate an early onset with a favourable prognosis (Lesser et al. 1960; Frazier, 1965; Theander, 1970; Halmi et al. 1973, 1976; Morgan \& Russell, 1975; Pierloot et al. 1975; Willi \& Hagemann, 1976; Sturzenberger et al. 1977; Hsu et al. 1979). A few authors (Tolstrup, 1965; Warren, 1968; Browning \& Miller, 1968; Garfinkel et al. 1977), on the other hand, report no prognostic distinction between illnesses of early and late onset. Seidensticker \& Tzagournis (1968) regard an onset up to the age of 30 as prognostically favourable, and Dally (1969) even claims that the prognosis of anorexia nervosa commencing before the age of 14 is unfavourable.

\section{(b) Hysterical personality structure}

This is seen as progressing with a favourable course by Lesser et al. (1960), Blitzer et al. (1961), Kay \& Shapira (1965), Rollins \& Blackwell (1968), Dally (1969), Kalucy et al. (1976) and Goetz et al. (1977).

\section{(c) Parent-child relationship}

Good relations between the patient and his/her parents are said to indicate a good outcome by several workers (Kay \& Shapira, 1965; Dally, 1969; Crisp et al. 1974; Morgan \& Russell, 1975; Hsu et al. 1979), but this is questioned by Pierloot et al. (1975) and Theander (1970).

\section{(d) Duration of symptoms}

A short history prior to hospitalization or a brief episode of illness augurs well according to Kay \& Shapira (1965), Dally \& Sargant (1966), Seidensticker \& Tzagournis (1968), Pierloot et al. (1975), Morgan \& Russell (1975) and Hsu et al. (1979). Browning \& Miller (1968), by contrast, regard this factor as insignificant.

\section{(e) Duration of in-patient treatment and number of readmissions}

These factors emerge as unconnected with the course of the condition from the work of Browning \& Miller (1968), Dally (1969) and Morgan \& Russell (1975), but suggest a good prognosis according to other studies (Seidensticker \& Tzagournis, 1968; Theander, 1970; Halmi et al. 1973, 1976; Garfinkel et al. 1977).

\section{(f) Socioeconomic status}

Anorexics from the upper social classes fared better in the studies of Seidensticker \& Tzagournis (1968), Halmi et al. (1973, 1976), Kalucy et al. (1976), Garfinkel et al. (1977) and Hsu et al. (1979), but carried no prognostic significance in the reports of Dally (1969) and Theander (1970).

\section{(g) Disturbances of body perception}

A diminution of these disturbances after weight increase has been reported as suggesting a good outcome by Slade \& Russell (1973), Garfinkel et al. (1977) and Kalucy et al. (1977). Slade \& Russell (1973) and Kalucy et al. (1977) report that a good outcome is related to a correction of such perceptual disturbances as a result of weight increase.

\section{(h) Hyperactivity and dieting}

The exclusive use of these methods to reduce weight is regarded as pointing to a favourable outcome by Beumont et al. (1976), but as unimportant prognostically by Halmi et al. $(1973,1976)$ and Pierloot et al. (1975). 


\section{(i) Specifically unfavourable prognostic factors}

These include vomiting during the onset and course of anorexia nervosa (Theander, 1970; Halmi et al. 1973; Crisp et al. 1974; Beumont et al. 1976; Garfinkel et al. 1977; Hsu et al. 1979); purgative abuse and bulimia during the onset and course of the condition (Theander, 1970; Halmi et al. 1973; Beumont et al. 1976; Willi \& Hagemann, 1976; Kalucy et al. 1976; Hsu et al. 1979); extreme loss of weight (Dally, 1969; Morgan \& Russell, 1975; Hsu et al. 1979); depressive and obsessive-compulsive symptoms (Halmi et al. 1973); chronicity, associated particularly with obsessive-compulsive personality traits (Lesser et al. 1960; Kay \& Shapira, 1965; Dally, 1969; Bhanji \& Thompson, 1974; Kalucy et al. 1976; Goetz et al. 1977); older maternal age (Dally, 1969; Theander, 1970; Halmi et al. 1973); premorbid developmental and clinical abnormal phenomena (Dally, 1969; Morgan \& Russell, 1975); high rates of physical complaints (Halmi et al. 1973; Stonehill \& Crisp, 1977); acute body perception disturbances (Garfinkel et al. 1977; Kalucy et al. 1977); neuroticism (Dally, 1969; Pierloot et al. 1975); psychological test-results suggestive of psychosis (Pierloot et al. 1975); the masculine sex (Kalucy et al. 1977); and marriage (Seidensticker \& Tzagournis, 1968; Kalucy et al. 1976; Hsu et al. 1979; except for the study of Willi \& Hagemann, 1976).

\section{CONCLUSIONS}

In toto, research on the course of anorexia nervosa does not constitute a unified whole. In addition to the numerous methodological problems, many of the conclusions are probably drawn from atypical samples. Furthermore, the proportion of dropouts during re-examination, which reaches a maximum figure of $77 \%$ with an average of $11 \%$, has not been subjected to a systematic test of trends in respect of individual characteristics. The extent to which certain findings are representative has therefore remained insufficiently analysed. It could be that the methods used for re-examination exercised a strong influence on the proportion of drop-outs. It is even possible that it is precisely the least reliable examinations - those relying on information obtained from telephone enquiries or from relatives - which actually lead to lower drop-out rates than structured examinations carried out in direct contact with the patient. The inadequate sampling of follow-up data is also indicated by the contradictory assessment of some prognostic factors. In view of the fact that most of these factors are described in relative isolation from one another, it cannot be determined which of them is the more significant.

In the light of these considerations we may formulate the needs of any future research dealing with the course of anorexia nervosa. First, the diagnostic classification of symptoms must be documented unequivocally in order to enable changes to be assessed and studies to be compared with one another. In addition, future studies should not rely on restrictive categories of patient populations.

It is necessary to record not only syndrome-specific variables but also aspects of general psychopathology and psychosocial adjustment, both at the onset as well as during the course of the illness. For these reasons, uni-dimensional follow-up studies which restrict themselves, for example, to the criterion of body weight are relatively useless. Furthermore, the criteria of psychosocial adjustment, if such are to be evaluated, must be operationalized so that crossvalidation and verification become feasible.

Whether they are retrospective or prospective, follow-up studies must specify not only the proportion of drop-outs but also ascertain whether these subjects differ in any essential respects from other patients and so make it possible to judge to what extent the studies are representative. Since the natural history of anorexia nervosa cannot be assessed in less than 4 years, it is necessary to observe patients for at least this period or to establish that it is possible to achieve an equally reliable forecast within a shorter time.

Follow-up results should be based on a direct re-examination and not on indirect questions to relatives and friends or on telephone calls. At the same time, the reliability of any procedures must be documented or demonstrated. For this purpose use should be made of standardized procedures, i.e. interviews and questionnaires. 
A multidimensional strategy of evaluation would facilitate an assessment of the determinants of prognosis. An adequate number of samples must be obtained, and the data should be analysed by appropriate multivariate techniques. Research into anorexia nervosa which employs these criteria will be in a better position than hitherto to report more accurately on the course of the condition.

\section{H.-C. STEINHAUSEN AND K. GLANVILLE}

\section{REFERENCES}

Andersen, A. E. (1977). Atypical anorexia nervosa. In Anorexia Nervosa (ed. R. A. Vigersky), pp. 11-19. Raven Press: New York.

Beck, J. C. \& Brachner-Mortensen, K. (1954). Observations on the prognosis in anorexia nervosa. Acta Medica Scandinavica 149, 409-430.

Beumont, P. J. V., George, G. C. W. \& Smart, D. E. (1976). 'Dieters' and 'vomiters and purgers' in anorexia nervosa. Psychological Medicine 6, 617-622.

Bhanji, S. \& Thompson, J. (1974). Operant conditioning in the treatment of anorexia nervosa. British Journal of Psychiatry 124, 166-172.

Blitzer, J. R., Rollins, N. \& Blackwell, A. (1961). Children who starve themselves: anorexia nervosa. Psychosomatic Medicine 23, 369-383.

Brady, J. P. \& Rieger, W. (1975). Behavioral treatment of anorexia nervosa. In Applications of Behavior Modification (ed. T. Thompson and W. S. Dockens III), pp. 45-63. Academic Press: New York.

Browning, C. H. \& Miller, S. I. (1968). Anorexia nervosa: a study in prognosis and management. American Journal of Psychiatry 124, $1128-1132$.

Bruch, H. (1973). Outcome and outlook. In Eating Disorders (ed. H. Bruch), pp. 377-387. Basic Books: New York.

Cantwell, D. P., Sturzenberger, S., Burroughs, J., Salkin, B. \& Green, J. (1977). Anorexia nervosa - an affective disorder? Archives of General Psychiatry 34, 1087-1093.

Cremerius, J. (1978). Zur Prognose der Anorexia nervosa. Zeitschrift für psychosomatische Medizin und Psychoanalyse 24, 56-69.

Crisp, A. H. (1965). Some aspects of the evolution, presentation and follow-up of anorexia nervosa. Proceedings of the Royal Society of Medicine 58, 814-820.

Crisp, A. H. (1966). A treatment regime for anorexia nervosa. British Journal of Psychiatry 112, 505-512.

Crisp, A. H., Harding, G. \& McGuiness, B. (1974). Anorexia nervosa. Psychoneurotic characteristics of parents : relationship to prognosis. Journal of Psychosomatic Research 18, 167-173.

Dally, P. J. (1969). Anorexia Nervosa. Heinemann Medical Books: London.

Dally, P. J. \& Sargant, W. (1966). Treatment and outcome of anorexia nervosa. British Medical Journal ii, 793-795.

Farquharson, R. F. \& Hyland, H. H. (1966). Anorexia nervosa: the course of 15 patients treated from 20 to 30 years previously. Canadian Medical Association Journal 96, 411-419.

Feighner, J., Robins, E., Guze, S., Woodruff, R., Winokur, G. \& Munoz, R. (1972). Diagnostic criteria for use in psychiatric research. Archives of General Psychiatry 26, 57-63.

Frahm, H. (1965). Ergebnisse einer systematisch durchgeführten somatisch orientierten Behandlungsform bei Kranken mit Anorexia nervosa. In Anorexia Nervosa (ed. J. E. Meyer and H. Feldman), pp. 64-70. Thieme: Stuttgart.

Frazier, S. H. (1965). Anorexia nervosa. Diseases of the Nervous System 26, 155-159.

Fries, H. (1977). Studies on secondary amenorrhea, anorectic behavior and body-image perception: importance for the early recognition of anorexia nervosa. In Anorexia Nervosa (ed. R. A. Vigersky), pp. 163-176. Raven Press: New York.

Garfinkel, P. E., Moldofsky, H. \& Garner, D. M. (1977). The outcome of anorexia nervosa: significance of clinical features, body image and behavior modification. In Anorexia Nervosa (ed. R. A. Vigersky), pp. 315-329. Raven Press: New York.

Goetz, P. L., Succop, R. A., Reinhart, J. B. \& Miller, A. (1977). Anorexia nervosa in children: a follow-up study. American Journal of Orthopsychiatry 47, 597-603.
Halmi, K. A., Brodland, G. \& Loney, J. (1973). Prognosis in anorexia nervosa. Annals of Internal Medicine 78, 907.909.

Halmi, K. A., Brodland, G. \& Rigas, C. (1976). A follow-up study of seventy nine patients with anorexia nervosa: an evaluation of prognostic factors and diagnostic criteria. Life History Research in Psychopathology, Vol. 4 (ed. R. Writ, G. Winokur and M. Roff), pp. 290-300. University of Minnesota Press: Minneapolis.

Hsu, L. K. G., Crisp, A. H. \& Harding, B. (1979). Outcome of anorexia nervosa. Lancet i, 61-65.

Kalucy, R. S., Crisp, A. H., Chard, T., McNeilly, A., Chen, C. N. \& Lacey, J.H. (1976). Nocturnal hormonal profiles in massive obesity, anorexia nervosa and normal females. Journal of Psychosomatic Research 20, 595-604.

Kalucy, R. S., Crisp, A. H. \& Harding, B. (1977). A study of 56 families with anorexia nervosa. British Journal of Medical Psychology 50, 381-395.

Kay, D. W. (1953). Anorexia nervosa: a study in prognosis. Proceedings of the Royal Society of Medicine 46, 669-674.

Kay, D. W. \& Shapira, K. (1965). The prognosis in anorexia nervosa. In Anorexia Nervosa (ed. J. E. Meyer and H. Feldman), pp. 113117. Thieme: Stuttgart.

Lesser, L. I., Ashenden, B. J., Debushey, M. \& Eisenberg, L. (1960). Anorexia nervosa in children. American Journal of Orthopsychiatry 30, 572-580.

Meyer, J. E. (1961). Das Syndrom der Anorexia nervosa. Katamnestische Untersuchungen. Archiv für Psychiatrie und Nervenkrankheiten 202, 31-59.

Morgan, H. G. \& Russell, G. F. M. (1975). Value of family background and clinical features as predictors of long-term outcome in anorexia nervosa: four-year follow-up study of $\mathbf{4 1}$ patients. Psychological Medicine 5, 355-371.

Niederhoff, H., Wiesler, B. \& Kuenzer, W. (1975). Somatisch orientierte Behandlung der Anorexia nervosa. Monatsschrift für Kinderheilkunde 123, 343-344.

Niskanen, P., Jääskeläinen, J. \& Achté, K. (1974). Anorexia nervosa, treatment results and prognosis. Psychiatria Fennica 257-263.

Pertschuk, M. J. (1977). Behavior therapy: extended follow-up. In Anorexia Nervosa (ed. R. A. Vigersky), pp. 305-314. Raven Press: New York.

Petzold, E. (1979). Familienkonfrontationstherapie bei Anorexia nervosa. Verlag für medizinische Psychologie Dr C. J. Hogrefe: Göttingen.

Pierloot, R. A., Wellens, W. \& Houben, M. E. (1975). Elements of resistance to a combined medical and psychotherapeutic program in anorexia nervosa. Psychotherapie-Psychosomatik 26, 101-117.

Rollins, N. \& Blackwell, A. (1968). The treatment of anorexia nervosa in children and adolescents. Stage I. Journal of Child Psychology and Psychiatry 9,81-91.

Rollins, N. \& Piazza, E. (1978). Diagnosis of anorexia nervosa. Journal of the American Academy of Child Psychiatry 17, 126-137.

Rollins, N. \& Piazza, E. (1981). Anorexia nervosa. A qualitative approach to follow-up. Journal of the American Academy of Child Psychiatry 20, 167-183.

Rosman, B. L., Minuchin, S., Liebman, R. \& Baker, L. (1976). Input and outcome of family therapy in anorexia nervosa. In Successful Psychotherapy (ed. J. L. Claghorn), pp. 128-139. Brunner/Mazel: New York.

Schütze, G. (1980), Anorexia Nervosa, pp. 121-131. Huber: Bern.

Seidensticker, J.\& Tzagournis, M. (1968). Anorexia nervosa - clinical features and long-term follow-up. Journal of Chronic Diseases 21, 366-367.

Silverman, J. A. (1974). Anorexia nervosa: clinical observations in a successful treatment plan. Journal of Pediatrics 84, 68-73. 
Slade. P. D. \& Russell, G. F. M. (1973). Awareness of body dimensions in anorexia nervosa: cross-sectional and longitudinal studies. Psychological Medicine 3, 188-199.

Sours, J. A. (1969). Anorexia nervosa: nosology, diagnosis, developmental patterns, and power-control dynamics. In Adolescence: Psychosocial Perspectives (ed. G. Caplan and S. Lebovici), pp. 185-212. Basic Books: New York.

Stonehill, E. \& Crisp, A. H. (1977). Psychoneurotic characteristics of patients with anorexia nervosa before and after treatment and at follow-up 4-7 years later. Journal of Psychosomatic Research 21, 187-193.

Sturzenberger, S., Cantwell, P. D., Burroughs, J., Salkin, B. \& Green, J. K. (1977). A follow-up study of adolescent psychiatric inpatients with anorexia nervosa. Journal of the American Academy of Child Psychiatry 16, 703-715.

Theander, S. (1970). Anorexia nervosa a psychiatric investigation of 94 female patients. Acta psychiatrica scandinavica Supp. 214.

Thomä, H. (1961). Anorexia Nervosa. Huber: Bern.
Tolstrup, K. (1965). Die Charakteristika der jüngeren Fälle von Anorexia nervosa. In Anorexia Nervosa (ed. J. E. Meyer and H. Feldman), pp. 51-59. Thieme: Stuttgart.

Valanne, E. H., Taipale, V., Larkio-Miettinen, A.-K., Morén, R. \& Aukee, M. (1972). Anorexia nervosa. A follow-up study. Psychiatria Fennica 265-269.

Warren, W. (1968). A study of anorexia nervosa in young girls. Journal of Child Psychology and Psychiatry 9, 27-40.

Willi, J. \& Hagemann, R. (1976). Langzeitverläufe von Anorexia nervosa. Schweizerische Medizinische Wochenschrift 106, 1459-1465.

Williams, E. (1958). Anorexia nervosa: a somatic disorder. British Medical Journal ii, 190-195.

Ziegler, R. \& Sours, J. (1968). A naturalistic study of patients with anorectic nervosa admitted to an university medical center. Comprehensive Psychialry 9, 644-651.

Ziolko, H. U. (1978). Zur Katamnese der Pubertätsmagersucht. Archiv für Psychiatrie und Nervenkrankheiten 225, 117-125. 